\title{
Synthesis, Characterization, and Marked Polymerization Selectivity Characteristics of Binuclear Phenoxyiminato Organozirconium Catalysts.
}

\author{
Michael R. Salata and Tobin J. Marks* \\ Department of Chemistry, Northwestern University, Evanston, Illinois \\ 60208-3113 \\ t-marks@northwestern.edu
}

\section{Supporting Information}

Materials and Methods. All manipulations of air-sensitive materials were performed with rigorous exclusion of oxygen and moisture in flamed Schlenk-type glassware on a dual manifold Schlenk line, or interfaced to a high-vacuum line ( $10^{-5}$ Torr), or in a nitrogen-filled Vacuum Atmospheres glove box with a high capacity recirculator $(<1$ ppm $\mathrm{O}_{2}$ ). Argon and ethylene (Matheson, polymerization grade) were purified by passage through a supported $\mathrm{MnO}$ oxygen-removal column and an activated Davison 4A molecular sieve column. Ether solvents were purified by distillation from $\mathrm{Na} / \mathrm{K}$ alloy/benzophenone ketyl. Hydrocarbon solvents were distilled under nitrogen from $\mathrm{Na} / \mathrm{K}$ alloy. All solvents for high-vacuum line manipulations were stored in vacuo over $\mathrm{Na} / \mathrm{K}$ alloy in Teflon-valve sealed bulbs. Deuterated solvents were obtained from Cambridge 
Isotope Laboratories (all $\geq 99$ atom \%D), were freeze pump- thaw degassed, dried over $\mathrm{Na} / \mathrm{K}$ alloy and were stored in resealable flasks. Other non-halogenated solvents were dried over $\mathrm{Na} / \mathrm{K}$ alloy, and halogenated solvents were distilled from $\mathrm{CaH}_{2}$ and stored over activated Davison 4A molecular sieves. The reagents 2,6-di-iso-propylaniline, and chlorotrimethylsilane were purchased from Aldrich and used as received. The comonomer 1-hexene (Aldrich) was dried over $\mathrm{CaH}_{2}$. The reagents $\mathrm{LiCH}_{2} \mathrm{TMS}$ and $\mathrm{ZrCl}_{4}$ (Aldrich) were sublimed before use. Methylaluminoxane (MAO, obtained as a $10 \%$ toluene solution from Aldrich) was dried under high vacuum for $24 \mathrm{~h}$ to remove excess volatile aluminum alkyls before use. The reagents $\mathrm{ZrCl}_{4}(\mathrm{THF})_{2}{ }^{1}$ and $\mathrm{Zr}\left\{3-{ }^{t} \mathrm{Bu}-2-\right.$ (O) $\left.\mathrm{C}_{6} \mathrm{H}_{3} \mathrm{CH}=\mathrm{N}\left(2,6-{ }^{i} \mathrm{Pr}_{2} \mathrm{C}_{6} \mathrm{H}_{3}\right)\right\} \mathrm{Cl}_{3}(\mathrm{THF})^{2}$ (FI-Zr1) were prepared according to literature procedures, however, for the latter, heating at reflux for $16 \mathrm{~h}$ was required for complete reaction.

Physical and Analytical Measurements. NMR spectra were recorded on Varian UNITYInova-500 (FT, 500 MHz, 1H; 125 MHz, 13C) or UNITYInova-400 (FT, 400 $\mathrm{MHz}, 1 \mathrm{H} ; 100 \mathrm{MHz}, 13 \mathrm{C})$. Chemical shifts for ${ }^{1} \mathrm{H}$ and ${ }^{13} \mathrm{C}$ spectra were referenced using internal solvent resonances and are reported relative to tetramethylsilane. NMR experiments on air-sensitive samples were conducted in Teflon valve-sealed sample tubes (J.Young). Elemental analyses were performed by Midwest Microlab, Indianapolis, Indiana. ${ }^{13} \mathrm{C}$ NMR assays of polymer microstructure were conducted in mixture (1:1 in volume) of 1,2,4-trichlorobezene (containing $0.1 \mathrm{M} \mathrm{Cr}(\mathrm{acac})_{3}$ solution) and 1,1,2,2tetrachloroethane- $d_{2}$ at $130^{\circ} \mathrm{C}$. Signals were assigned according to the literature for polyethylene and ethylene $+\alpha$-olefin copolymers. ${ }^{3}$ Melting temperatures of polymers 
were measured by DSC (DSC 2920, TA Instruments, Inc.) from the second scan with a heating rate of $10^{\circ} \mathrm{C} / \mathrm{min}$. GPC measurements were performed on a Polymer Laboratories PL-GPC 220 instrument using 1,2,4-trichlorobenzene solvent (stabilized with $125 \mathrm{ppm}$ BHT) at $150{ }^{\circ} \mathrm{C}$. A set of three PLgel $10 \mu \mathrm{m}$ mixed columns was used. Samples were prepared at $160{ }^{\circ} \mathrm{C}$. Molecular weights were determined by GPC using narrow polystyrene standards and are not corrected. Laser desorption mass spectra were obtained with a Perseptive BioSystems time-of-flight MALDI mass spectrometer using a dithranol matrix.

\section{Synthesis of 2,7-di(2,6-diisopropylphenyl)imino-1,8-dihydroxynaphthalene (1).}

Under $\mathrm{N}_{2}$, the reagent 2,7-diformyl-1,8-dihydroxynaphthalene (2.15 g, $\left.9.93 \mathrm{mmol}\right)$ was dissolved in $50 \mathrm{~mL}$ dry dichloromethane. Activated molecular sieves $(2 \mathrm{~g})$ were then added, followed by formic acid (5 drops), and 2,6-di-iso-propylaniline ( $8.0 \mathrm{~mL}, 42$ mmol). The reaction was heated under reflux for $3 \mathrm{~h}$ during which time the 2,7-diformyl1,8-dihydroxynaphthalene gradually dissolved, and the reaction mixture color changed from yellow to dark red. The reaction mixture was then cooled and filtered. The volatiles were removed from the filtrate in vacuo, and the resulting red oily solid was washed with hexanes $(3 \times 15 \mathrm{~mL})$ and collected by filtration to give a pure orange solid. $\mathrm{X}$-ray quality single crystals were obtained by slow evaporation of a dichloromethane solution. Yield, $4.5 \mathrm{~g}(86 \%)$.

${ }^{1} \mathrm{H}$ NMR $\left(\mathrm{CDCl}_{3}, 23^{\circ} \mathrm{C}, 499.447 \mathrm{MHz}\right): \delta 14.56(\mathrm{~s}, \mathrm{OH}), 13.48(\mathrm{~s}, \mathrm{OH}), 8.75$ (s), 8.34

(s), $7.66(\mathrm{~d}, \mathrm{~J}=11.3 \mathrm{~Hz}), 6.78-7.35(\mathrm{~m}), 3.08\left(\mathrm{~d}, \mathrm{CHMe}_{2}\right), 1.20(\mathrm{~d}, \mathrm{CHMe})_{2}$. 
Anal. Found: C, 80.84; H, 7.90; N, 5.25. Calcd. for $\mathrm{C}_{36} \mathrm{H}_{42} \mathrm{~N}_{2} \mathrm{O}_{2}: \mathrm{C}, 80.86 ; \mathrm{H}, 7.92 ; \mathrm{N}$, 5.24.

\section{Synthesis of 2,7-Di(2,6-diisopropylphenyl)imino-1,8-di(trimethylsiloxy)naphthalene}

(2). The reagents 2,7-di(2,6-diisopropylphenyl)imino-1,8-dihydroxynapthalene (589 mg, $1.10 \mathrm{mmol})$ and $\mathrm{LiCH}_{2} \mathrm{TMS}(228.2 \mathrm{mg}, 2.424 \mathrm{mmol})$ were combined in a reaction flask in the glovebox. On the vacuum line, dry THF $(25 \mathrm{~mL})$ was added at $-78^{\circ} \mathrm{C}$ and the mixture was stirred for $30 \mathrm{~min}$, followed by stirring for $30 \mathrm{~min}$ at $0^{\circ} \mathrm{C}$. The volatiles were next removed in vacuo, and the resulting solid was dissolved in $20 \mathrm{~mL}$ dry dichloromethane. Next, chlorotrimethylsilane $(0.35 \mathrm{~mL}, 2.7 \mathrm{mmol})$ was added with stirring, and the resulting solution became hazy over a period of $4.5 \mathrm{~h}$. The reaction mixture was then filtered, and volatiles were removed from the filtrate in vacuo. Pure orange product was obtained by washing the resulting orange/brown solid with cold pentane $(5 \mathrm{~mL})$. X-ray quality single crystals were obtained by cooling a saturated pentane solution at $-10^{\circ} \mathrm{C}$. Yield, $598 \mathrm{mg}(80 \%)$.

${ }^{1} \mathrm{H} \mathrm{NMR}\left(\mathrm{CDCl}_{3}, 23{ }^{\circ} \mathrm{C}, 499.447 \mathrm{MHz}\right): \delta 8.762(\mathrm{~s}, 2 \mathrm{H}, H \mathrm{C}=\mathrm{N}), 8.475\left(\mathrm{~d}, 2 \mathrm{H},{ }^{3} \mathrm{~J}_{\mathrm{H}-\mathrm{H}}=\right.$ $8.457 \mathrm{~Hz}$, naph C3), $7.639\left(\mathrm{~d}, 2 \mathrm{H},{ }^{3} \mathrm{~J}_{\mathrm{H}-\mathrm{H}}=8.641 \mathrm{~Hz}\right.$, naph C4), $7.297\left(\mathrm{~d}, 2 \mathrm{H},{ }^{3} \mathrm{~J}_{\mathrm{H}-\mathrm{H}}=\right.$ $7.537 \mathrm{~Hz}, \operatorname{dipp} p), 7.231\left(\mathrm{t}, 4 \mathrm{H},{ }^{3} \mathrm{~J}_{\mathrm{H}-\mathrm{H}}=7.354 \mathrm{~Hz}, \operatorname{dipp} m\right), 3.152\left(\operatorname{sept}, 4 \mathrm{H},{ }^{3} \mathrm{~J}_{\mathrm{H}-\mathrm{H}}=6.802\right.$

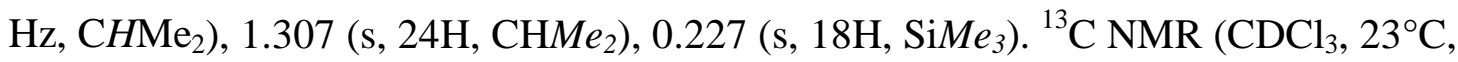
$100.745 \mathrm{MHz}) \delta 158.447,154.442,150.080,140.512,138.032,125.771,124.647$, $124.214,123.351,123.267,122.460,28.111,24.214,23.564,0.387$.

Anal. Found: C, 74.26; H, 8.60; N, 4.15. Calcd. for $\mathrm{C}_{36} \mathrm{H}_{42} \mathrm{~N}_{2} \mathrm{O}_{2}: \mathrm{C}, 74.28 ; \mathrm{H}, 8.61 ; \mathrm{N}$, 4.13. 


\section{Synthesis of $\left\{1,7-(\mathrm{O})_{2} \mathrm{C}_{10} \mathrm{H}_{4}-2,7-\left[\mathrm{CH}=\mathrm{N}\left(2,6-i \mathrm{Pr}_{2} \mathrm{C}_{6} \mathrm{H}_{3}\right)\right]_{2}\right\} \mathrm{Zr}_{2} \mathrm{Cl}_{6}(\mathrm{THF})_{2}\left(\mathrm{FI}^{2}-\mathrm{Zr}_{2}\right)$.}

Under $\mathrm{N}_{2}$, the reagent 2,7-di(2,6-diisopropylphenyl)imino-1,8-

di(trimethylsiloxy)naphthalene (484 $\mathrm{mg}, 0.713 \mathrm{mmol}$ ) in $20 \mathrm{~mL}$ dry dichloromethane was added dropwise with stirring to a solution of $\mathrm{ZrCl}_{4}(\mathrm{THF})_{2}(538 \mathrm{mg}, 1.43 \mathrm{mmol})$ in $20 \mathrm{~mL}$ dry dichloromethane at $-78^{\circ} \mathrm{C}$. The solution was then allowed to warm to room temperature and stirred for $1 \mathrm{~h}$ before heating to reflux for $24 \mathrm{~h}$. The volatiles were then removed in vacuo, and the residue was redissolved in $25 \mathrm{~mL}$ dichloromethane and filtered. Next, $20 \mathrm{~mL}$ dry toluene was added to the filtrate, and the resulting pure orange product was isolated by filtration. Attempts at isolating single crystals of $\mathrm{FI}^{2}-\mathrm{Zr}_{2}$ using standard methods gave powders. Yield, $762 \mathrm{mg}$ (79\%).

${ }^{1} \mathrm{H}$ NMR $\left(\mathrm{CD}_{2} \mathrm{Cl}_{2}, 23^{\circ} \mathrm{C}, 499.447 \mathrm{MHz}\right): \delta 12.953(\mathrm{~s}, 2 \mathrm{H}, H \mathrm{C}=\mathrm{N}), 8.162(\mathrm{~d}, 2 \mathrm{H}$,

${ }^{3} \mathrm{~J}_{\mathrm{H}-\mathrm{H}}=14.668 \mathrm{~Hz}$, naph C3), $7.620\left(\mathrm{~d}, 2 \mathrm{H},{ }^{3} \mathrm{~J}_{\mathrm{H}-\mathrm{H}}=8.149 \mathrm{~Hz}\right.$, naph C4), $7.501\left(\mathrm{t}, 2 \mathrm{H},{ }^{3} \mathrm{~J}_{\mathrm{H}-\mathrm{H}}\right.$ $=7.335 \mathrm{~Hz}, \operatorname{dipp} p), 7.330\left(\mathrm{~m}, 4 \mathrm{H},{ }^{3} \mathrm{~J}_{\mathrm{H}-\mathrm{H}}=8.964 \mathrm{~Hz}, \operatorname{dipp} m\right), 3.193\left(\mathrm{sept}, 4 \mathrm{H},{ }^{3} \mathrm{~J}_{\mathrm{H}-\mathrm{H}}=\right.$ $6.520 \mathrm{~Hz}, \mathrm{CHMe} 2), 1.286\left(\mathrm{~s}, 24 \mathrm{H},{ }^{3} \mathrm{~J}_{\mathrm{H}-\mathrm{H}}=6.519 \mathrm{~Hz}, \mathrm{CHMe}\right) .{ }^{13} \mathrm{C} \mathrm{NMR}\left(\mathrm{CD}_{2} \mathrm{Cl}_{2}, 23^{\circ} \mathrm{C}\right.$, 100.745 MHz): $\delta$ 171.281, 167.466, 147.524, 144.200, 142.764, 136.481, 134.094, $130.865,124.656,119.707,110.889,29.032,23.436$.

Anal. Found: C, 49.68; H, 5.48; N, 2.65. Calcd. for $\mathrm{C}_{36} \mathrm{H}_{42} \mathrm{~N}_{2} \mathrm{O}_{2}:$ C, 49.29; H, 5.26; N, 2.61 .

MALDI-TOF-MS: m/z 1074.2 (parent ion calcd 1070.04), 921.9 (-2 THF calcd 925.93), 731.8 (-2 THF and $-\mathrm{ZrCl}_{3}$ calcd 730.13). 
Ethylene Homopolymerization Experiments. On the high vacuum line $\left(10^{-5}\right.$ Torr $)$, ethylene polymerizations were carried out in $250 \mathrm{~mL}$ round-bottom three-neck flasks equipped with a large magnetic stirring bar, Morton indentations, and a thermocouple probe. In a typical experiment, a pre-assembled, degassed reactor containing toluene (50 $\mathrm{mL}$ ) and MAO (1000 eq relative to $\mathrm{Zr}$ ) was pre-saturated under of ethylene (1.0 atm) and equilibrated at the desired reaction temperature using an external bath. The catalyticallyactive species was freshly generated in toluene $(4.0 \mathrm{~mL})$ and MAO (500 equiv relative to metal). After an activation time ( $\sim 30 \mathrm{~min})$, the catalyst solution was then quickly injected into the rapidly stirred flask using a gas-tight syringe equipped with a flattened spraying needle. The temperature of the toluene solution was monitored in representative experiments using a thermocouple (OMEGA Type K thermocouple with a Model HH21 microprocessor thermometer). The reaction exotherm temperature rise was invariably less than $5^{\circ} \mathrm{C}$ during these polymerizations. After a measured time interval, the polymerization was quenched by the addition of $2 \%$ acidified methanol $(15 \mathrm{~mL})$. More methanol $(100 \mathrm{~mL})$ was then added, and the polymer was collected by filtration, washed with methanol and $10 \%$ aqueous $\mathrm{HCl}$, and dried on the high vacuum line overnight to a constant weight.

Ethylene + Olefin Copolymerization Experiments. On the high vacuum line $\left(10^{-5}\right.$ Torr), ethylene polymerizations were carried out in $250 \mathrm{~mL}$ round-bottom three-neck flasks equipped with a large magnetic stirring bar, Morton indentations, and a thermocouple probe. In a typical experiment, $10 \mathrm{~mL}$ of comonomer was vacuumtransferred into a graduated flask at $-78^{\circ} \mathrm{C}$, then the comonomer was injected into a pre- 
assembled, degassed reactor containing toluene $(50 \mathrm{~mL})$ and MAO (500 equiv relative to $\mathrm{Zr})$. The mixture was pre-saturated under of ethylene $(1.0 \mathrm{~atm})$ and equilibrated at the desired reaction temperature using an external bath. The catalytically-active species was freshly generated in toluene $(4.0 \mathrm{~mL})$ and $\mathrm{MAO}$ (500 eq relative to metal). After an activation time ( $\sim 30 \mathrm{~min})$, the catalyst solution was then quickly injected into the rapidly stirred flask using a gas-tight syringe equipped with a flattened spraying needle. The temperature of the toluene solution was monitored in representative experiments using a thermocouple (OMEGA Type K thermocouple with a Model HH21 microprocessor thermometer). The reaction exotherm temperature rise was invariably less than $5^{\circ} \mathrm{C}$ during these polymerizations. After a measured time interval, the polymerization was quenched by the addition of $2 \%$ acidified methanol $(15 \mathrm{~mL})$. More methanol $(100 \mathrm{~mL})$ was then added, and the polymer was collected by filtration, washed with methanol and $10 \%$ aqueous $\mathrm{HCl}$, and dried on the high vacuum line overnight to a constant weight.

\section{Crystal Structures of 1 and 2.}

\section{A. 2,7-Di(2,6-diisopropylphenyl)imino-1,8-dihydroxynaphthalene (1). A} summary of crystal structure data for compound $\mathbf{1}$ is presented in Table S1. Selected bond distances and angles for $\mathbf{1}$ are summarized in Table S2. Figure S1 shows the solid state structure of the binucleating ligand precursor $\mathbf{1}$. The asymmetric crystal structure reveals that $\mathrm{O}(2)$ actually is in essence deprotonated by the proximate imine functionality $\mathrm{N}(2)$. A proton was located in the difference map between $\mathrm{O}(1)$ and $\mathrm{O}(2)$ in a pseudochelating fashion which causes the anilino group to assume a trans geometry due to the 
steric demand of the isopropyl groups. The crystal structure explains the asymmetry and room temperature broadness of the ${ }^{1} \mathrm{H}$ NMR spectrum.

\section{B. 2,7-Di(2,6-diisopropylphenyl)imino-1,8-di(trimethylsiloxy)naphthalene}

(2). A summary of crystal structure data for compound $\mathbf{2}$ is presented in Table $\mathrm{S} 1$.

Selected bond distances and angles for $\mathbf{2}$ are summarized in Table S3. Figure S2 shows the solid state structure of $\mathbf{2}$. The crystal structure reveals the symmetric nature of the molecule, and as expected, the bulky trimethylsiloxy groups have close nonbonded contacts with the di-isopropylanilino groups.

\section{References}

${ }^{1}$ Manzer, L. E. Inorg. Synth. 1982, 21, 136.

${ }^{2}$ Pennington, D. A.; Clegg, W.; Coles,S. J.; Harrington, R. W.; Hursthouse, M. B.;

Hughes, D. L.; Light, M. E.; Schormann, M.; Bochmann, M.; Lancaster, S. J. Dalton

Trans. 2005, 3, 561-571.

${ }^{3}$ Pooter, M. De.; Smith, P. B.; Dohrer, K. K.; Bennett, K. F.; Meadows, M. D.; Smith, C. G.; Schouwenaars, H. P.; Geerards, R. A. J. App. Polym. Sci. 1991, 42, 399-408. 


\section{Figure Captions}

Figure S1. ORTEP plot and atom numbering scheme for 2,7-di(2,6-diisopropylphenyl)imino-1,8-dihydroxynaphthalene (1). Thermal ellipsoids are drawn at the $50 \%$ probability level.

Figure S2. ORTEP plot and numbering scheme for 2,7-di(2,6-diisopropylphenyl)imino1,8-di(trimethylsiloxy)naphthalene (2). Thermal ellipsoids are drawn at the 50\% probability level.

Figure S3. ${ }^{1} \mathrm{H}$ NMR spectrum $\left(\mathrm{CDCl}_{3}, 500 \mathrm{MHz}\right)$ of 2,7-di(2,6-diisopropylphenyl)imino1,8-dihydroxynaphthalene (1).

Figure S4. ${ }^{1} \mathrm{H}$ NMR spectrum $\left(\mathrm{CDCl}_{3}, 400 \mathrm{MHz}\right)$ of 2,7-di(2,6-diisopropylphenyl)imino1,8-dihydroxynaphthalene $(\mathbf{1})$ at $-33^{\circ} \mathrm{C}$.

Figure S5. ${ }^{1} \mathrm{H}$ NMR spectrum $\left(\mathrm{CDCl}_{3}, 500 \mathrm{MHz}\right)$ of 2,7-di(2,6-diisopropylphenyl)imino1,8-di(trimethylsiloxy)naphthalene (2).

Figure S6. ${ }^{13} \mathrm{C}$ NMR spectrum $\left(\mathrm{CDCl}_{3}, 100 \mathrm{MHz}\right)$ of 2,7-di(2,6-diisopropylphenyl)imino1,8-di(trimethylsiloxy)naphthalene (2).

Figure S7. ${ }^{1} \mathrm{H}$ NMR spectrum $\left(\mathrm{CD}_{2} \mathrm{Cl}_{2}, 500 \mathrm{MHz}\right)$ of $\left\{1,7-(\mathrm{O})_{2} \mathrm{C}_{10} \mathrm{H}_{4}-2,7-[\mathrm{CH}=\mathrm{N}(2,6-\right.$ $\left.\left.\left.i \mathrm{Pr}_{2} \mathrm{C}_{6} \mathrm{H}_{3}\right)\right]_{2}\right\} \mathrm{Zr}_{2} \mathrm{Cl}_{6}(\mathrm{THF})_{2}\left(\mathbf{F I}^{\mathbf{2}}-\mathbf{Z r}_{2}\right)$.

Figure S8. ${ }^{13} \mathrm{C}$ NMR spectrum $\left(\mathrm{CD}_{2} \mathrm{Cl}_{2}, 100 \mathrm{MHz}\right)$ of $\left\{1,7-(\mathrm{O}){ }_{2} \mathrm{C}_{10} \mathrm{H}_{4}-2,7-[\mathrm{CH}=\mathrm{N}(2,6-\right.$ $\left.\left.\left.i \mathrm{Pr}_{2} \mathrm{C}_{6} \mathrm{H}_{3}\right)\right]_{2}\right\} \mathrm{Zr}_{2} \mathrm{Cl}_{6}(\mathrm{THF})_{2}\left(\mathbf{F I}^{\mathbf{2}}-\mathbf{Z r}_{2}\right)$.

Figure 9. MALDI mass spectrum of $\left\{1,7-(\mathrm{O})_{2} \mathrm{C}_{10} \mathrm{H}_{4}-2,7-[\mathrm{CH}=\mathrm{N}(2,6-\right.$ $\left.\left.\left.i \mathrm{Pr}_{2} \mathrm{C}_{6} \mathrm{H}_{3}\right)\right]_{2}\right\} \mathrm{Zr}_{2} \mathrm{Cl}_{6}(\mathrm{THF})_{2}\left(\mathbf{F I}^{\mathbf{2}}-\mathbf{Z r}_{2}\right)$ with dithranol as the supporting matrix. 
Figure S1.

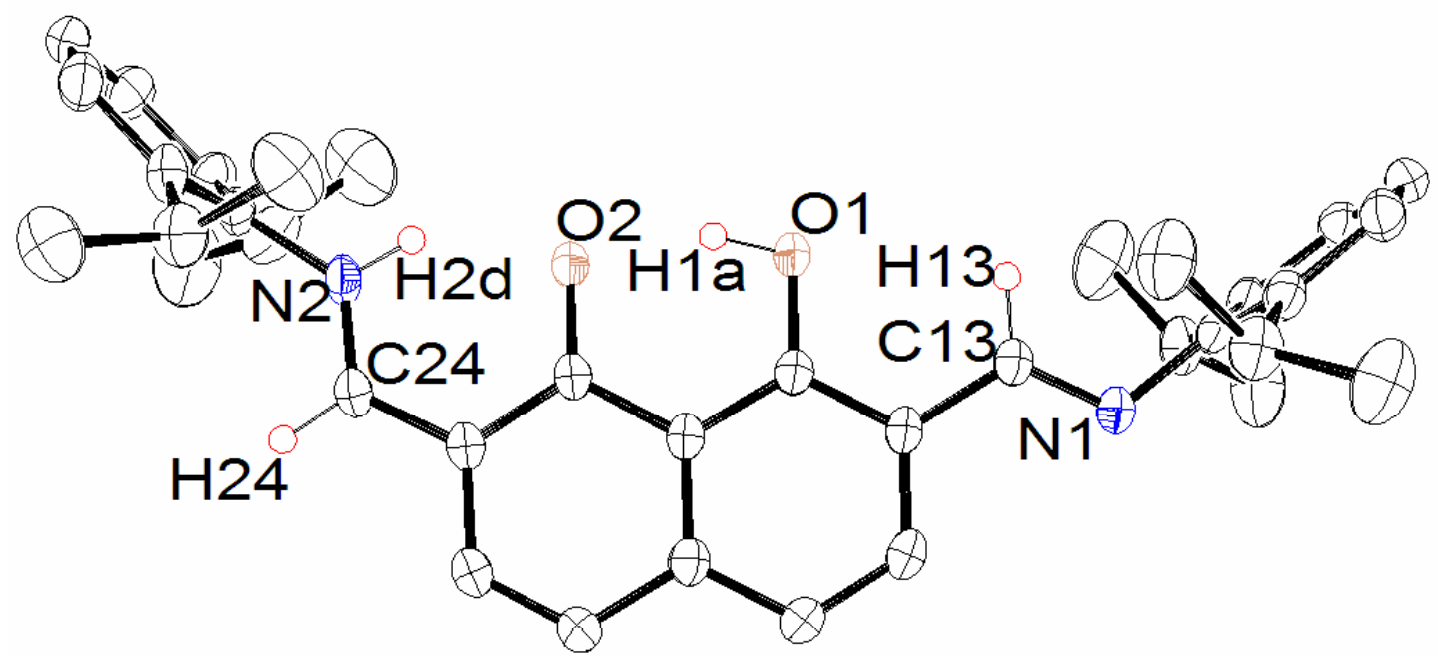

Figure S2.

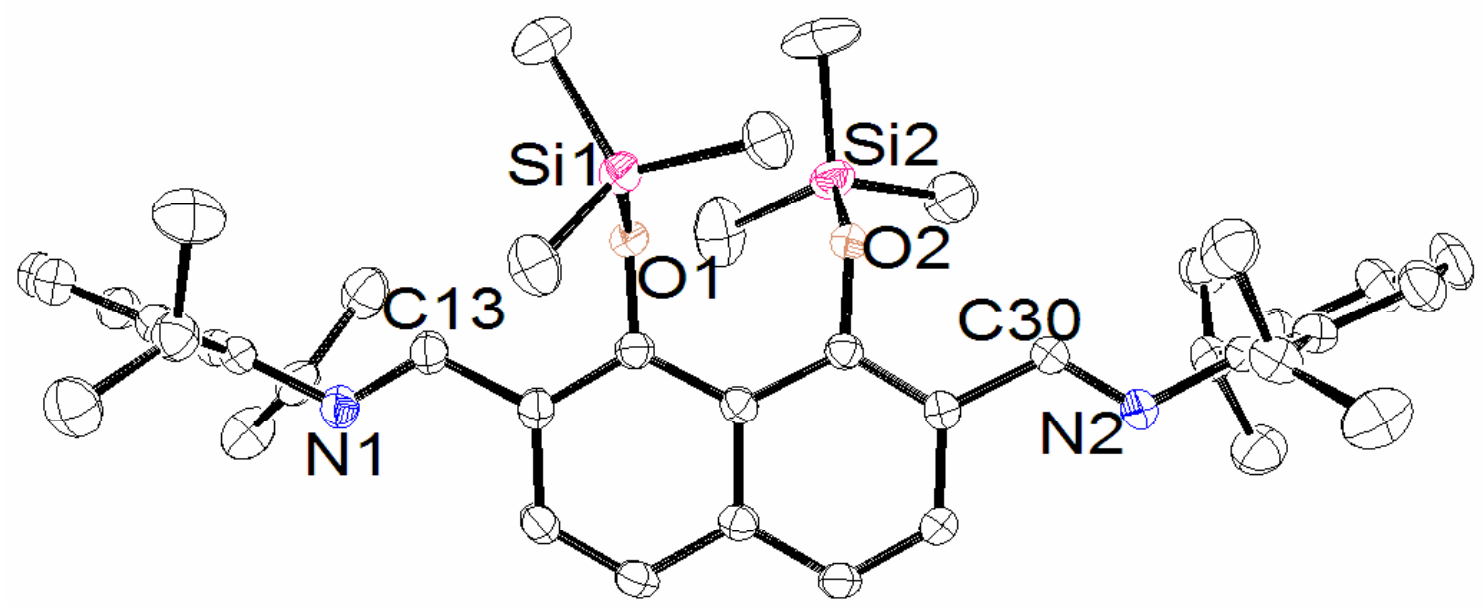


Figure S3.

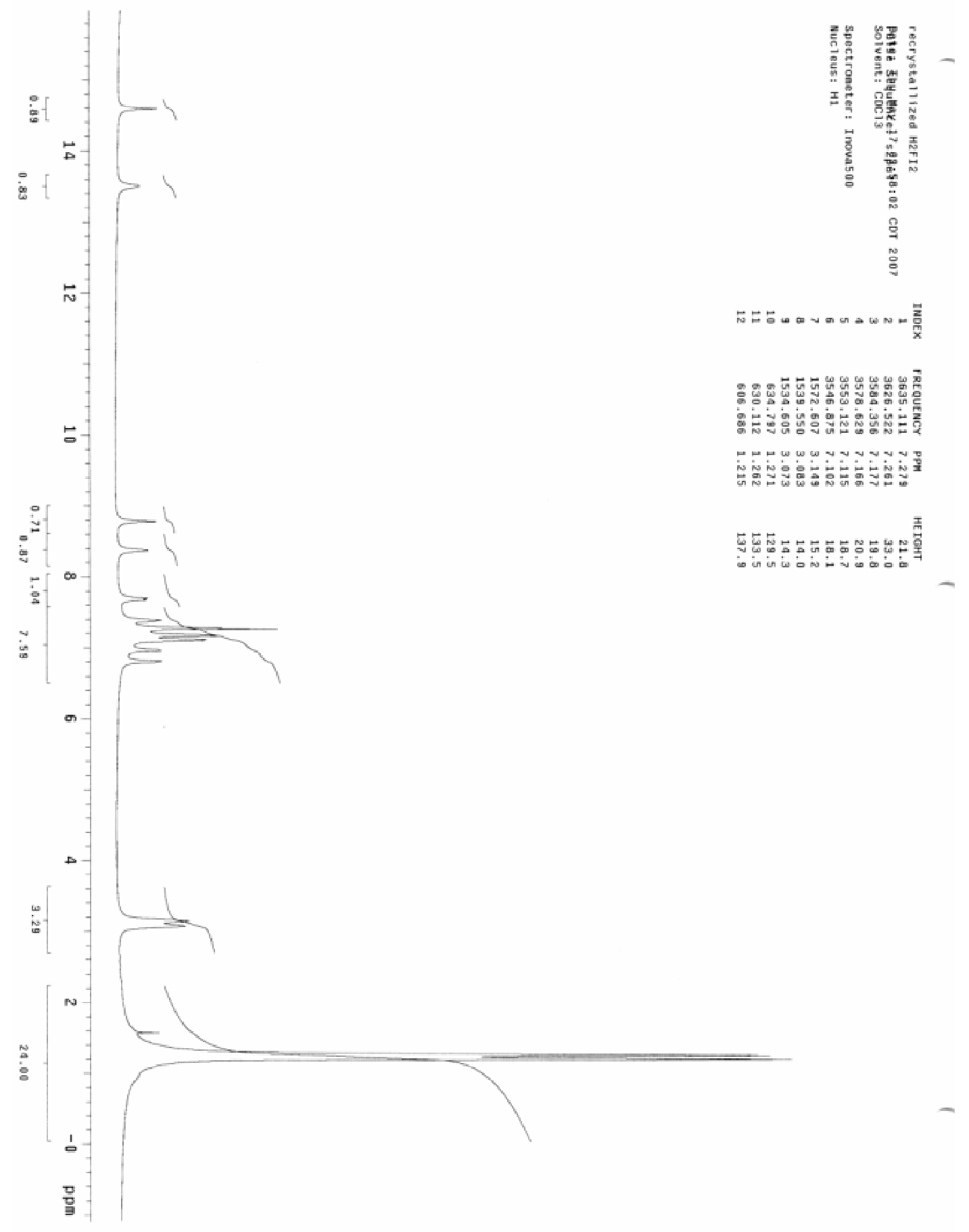


Figure S4.

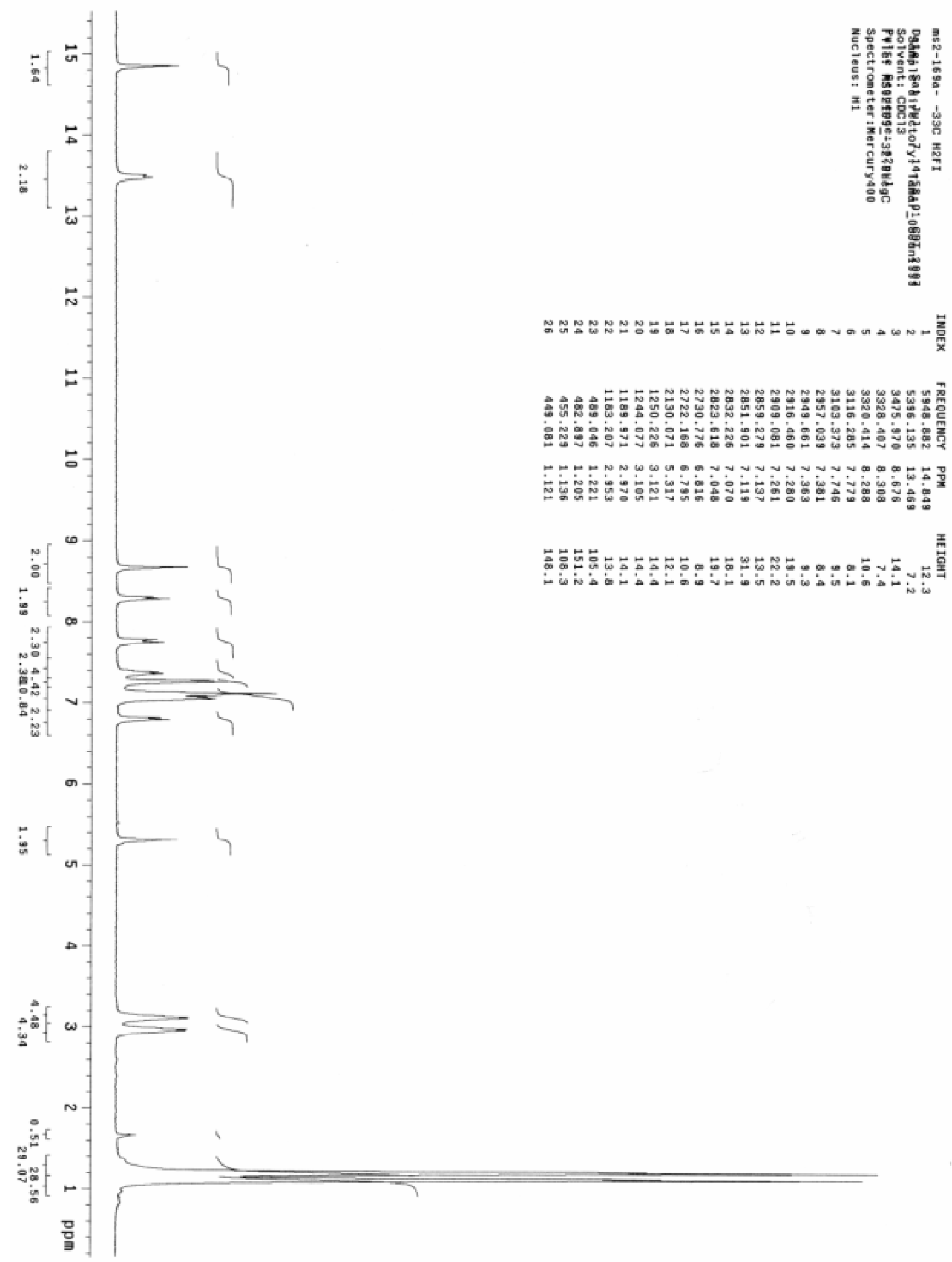


Figure S5.

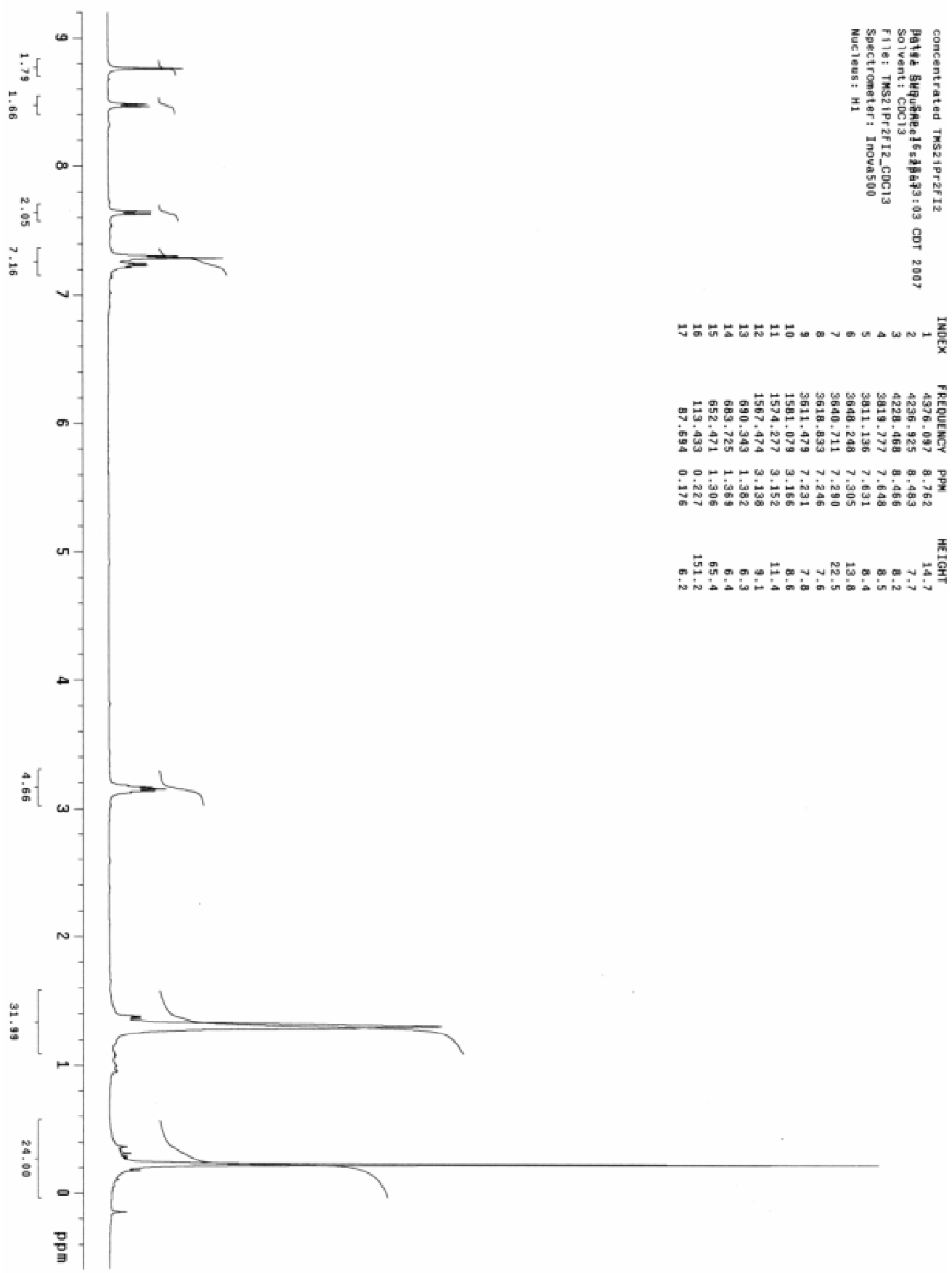


Figure S6.

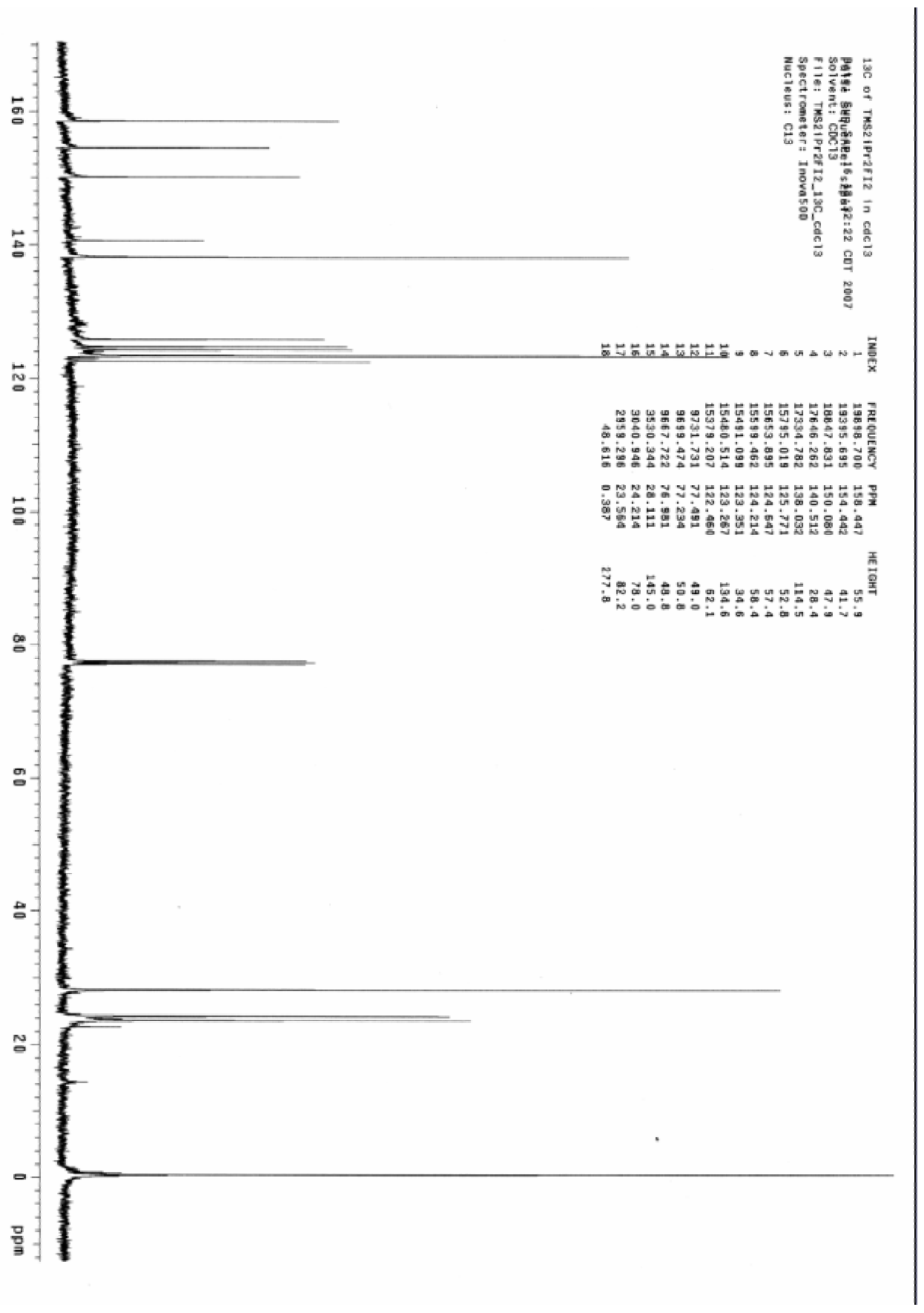


Figure S7.

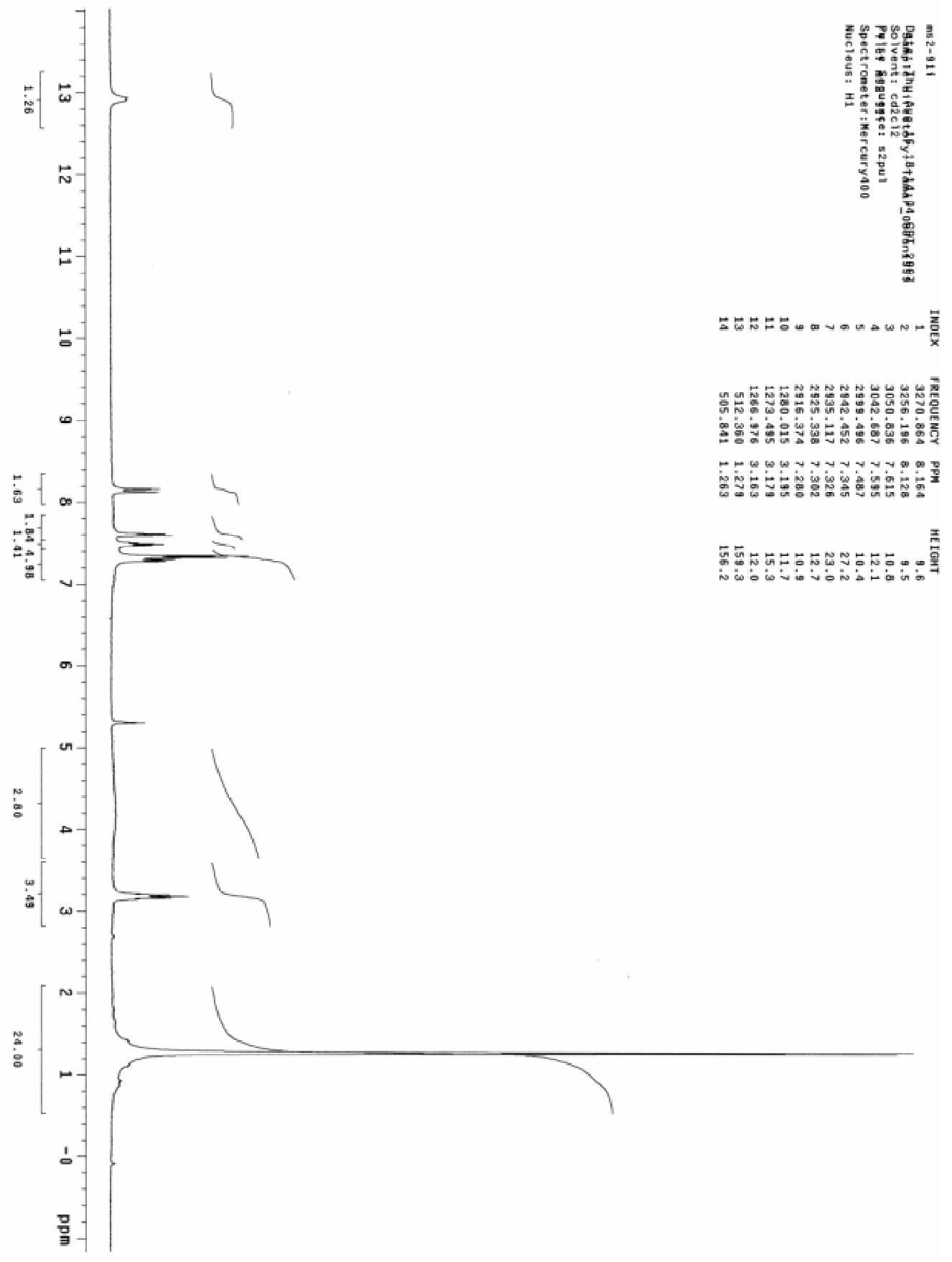


Figure S8.

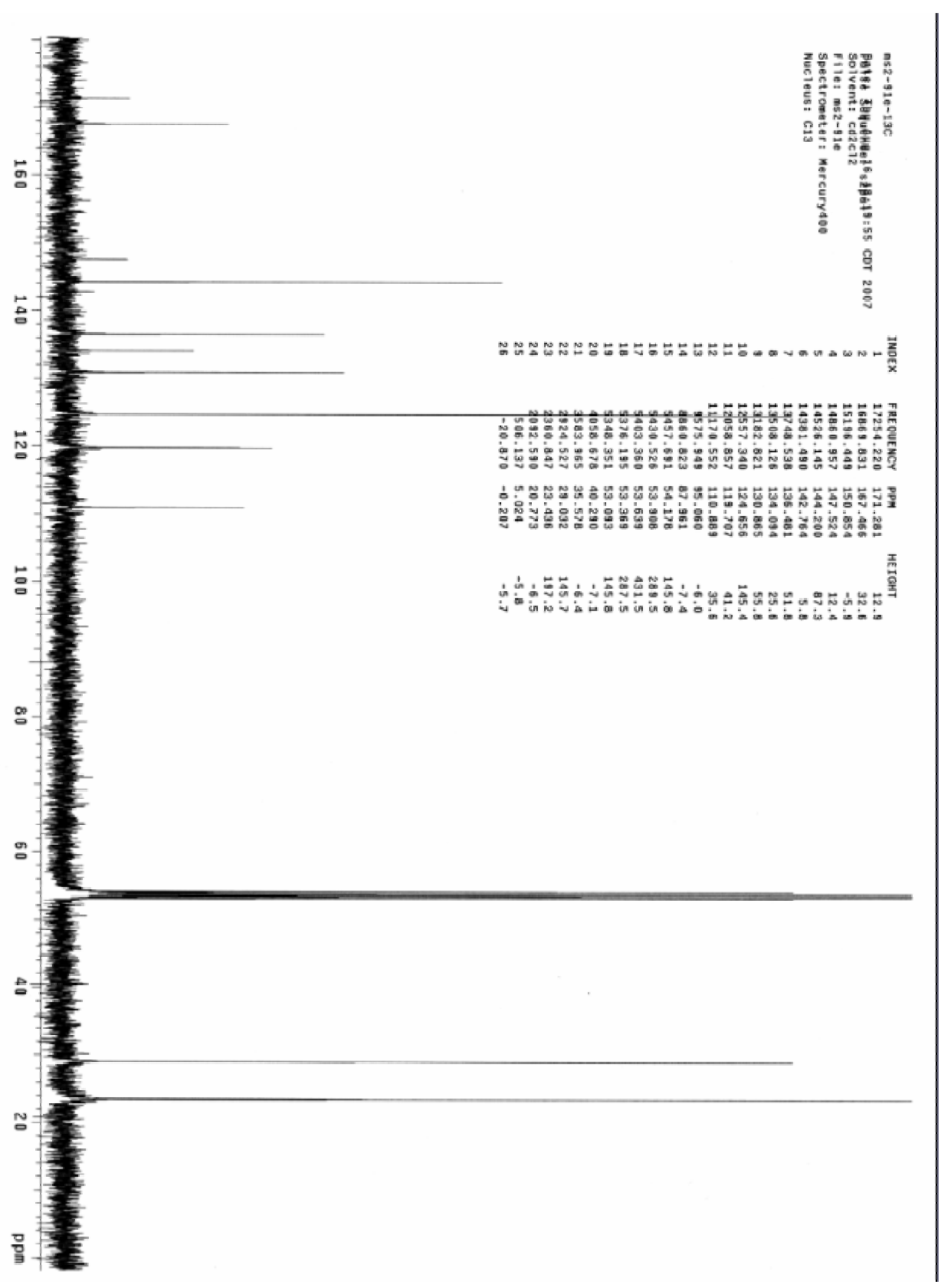




\section{Figure S9.}

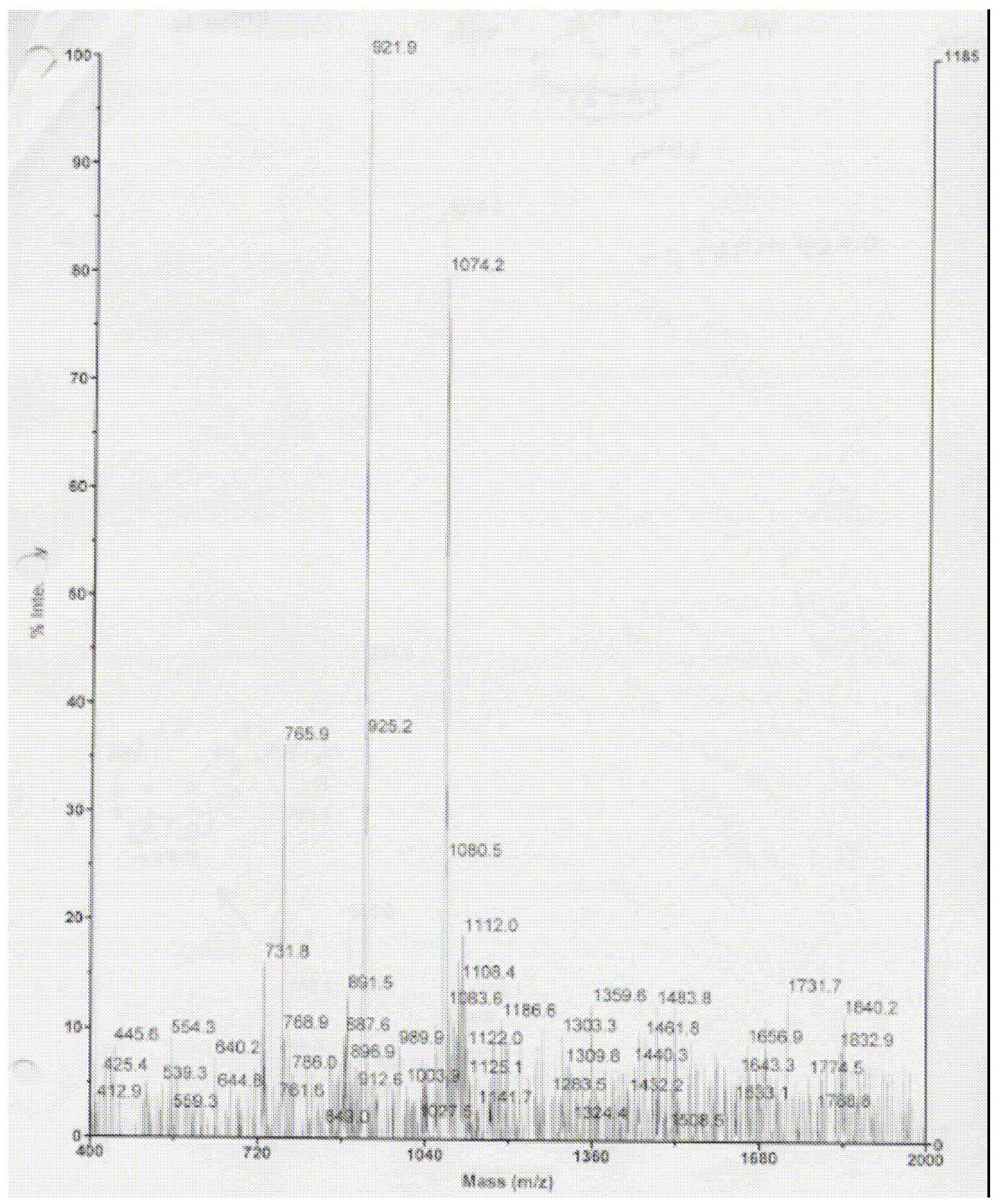

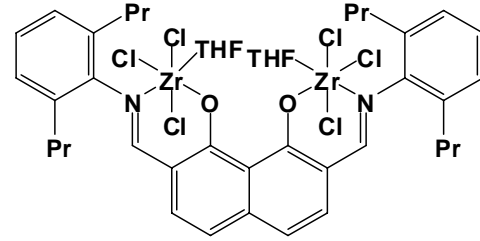

Chemical Formula: $\mathrm{C}_{44} \mathrm{H}_{54} \mathrm{Cl}_{6} \mathrm{~N}_{2} \mathrm{O}_{4} \mathrm{Zr}_{2}$ Molecular Weight: 1070.08
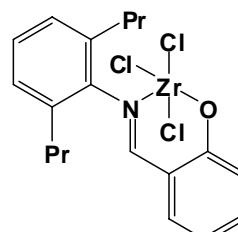

Chemical Formula: $\mathrm{C}_{36} \mathrm{H}_{40} \mathrm{Cl}_{6} \mathrm{~N}_{2} \mathrm{O}_{2} \mathrm{Zr}$ Exact Mass: 921.93

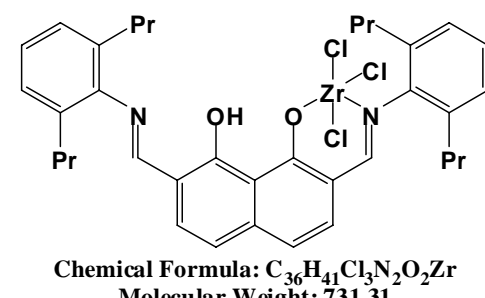

Molecular Weight: 731.31 
Table 1. Summary of the Crystal Structure Data for Complexes 1 and 2.

\begin{tabular}{|c|c|c|}
\hline Complex & 1 & 2 \\
\hline Formula & $\mathrm{C}_{36} \mathrm{H}_{42} \mathrm{~N}_{2} \mathrm{O}_{2}$ & $\mathrm{C}_{42} \mathrm{H}_{58} \mathrm{~N}_{2} \mathrm{O}_{2} \mathrm{Si}_{2}$ \\
\hline Formula Weight & 534.72 & 679.08 \\
\hline Crystal Dimensions (mm) & $0.506 \times 0.326 \times 0.186$ & $0.598 \times 0.232 \times .070$ \\
\hline Crystal System & Monoclinic & Monoclinic \\
\hline$a, \AA$ & $10.0592(8)$ & $26.914(6)$ \\
\hline$b, \AA$ & $23.0731(18)$ & $9.765(2)$ \\
\hline$c, \AA$ & $13.9928(11)$ & $16.289(4)$ \\
\hline$\alpha, \operatorname{deg}$ & 90 & 90 \\
\hline$\beta, \operatorname{deg}$ & $109.4350(10)$ & 109.311(3) \\
\hline$\gamma, \mathrm{deg}$ & 90 & 90 \\
\hline $\mathrm{V}, \AA^{\check{3}}$ & $3062.6(4)$ & $4040.1(15)$ \\
\hline Space group & $\mathrm{P} 2(1) / \mathrm{n}$ & $\mathrm{Cc}$ \\
\hline$Z$ value & 4 & 4 \\
\hline $\mathrm{D}_{\text {calc }}, \mathrm{mg} / \mathrm{m}^{3}$ & 1.160 & 1.116 \\
\hline Temp K & $153(2)$ & $153(2)$ \\
\hline$\mu, \mathrm{mm}^{-1}$ & 0.071 & 0.123 \\
\hline Radiation & $\operatorname{MoK} \alpha$ & $\operatorname{MoK} \alpha$ \\
\hline $2 \theta$ range, deg & 1.77 to 28.91 & 1.60 to 28.78 \\
\hline Intensities (unique, $\left(\mathrm{R}_{\text {int }}\right)$ & $7436,0.0299$ & $9137,0.0828$ \\
\hline $\mathrm{R}$ & 0.0616 & 0.0462 \\
\hline wR2 & 0.1614 & 0.1123 \\
\hline
\end{tabular}


Table 2. Selected Bond Distances (Å) and Angles (deg) for compound 1.

\begin{tabular}{|c|c|c|c|}
\hline \multicolumn{4}{|c|}{ Distances } \\
\hline $\mathrm{O}(2)-\mathrm{C}(20)$ & $1.289(2)$ & $\mathrm{N}(2)-\mathrm{C}(25)$ & $1.438(2)$ \\
\hline $\mathrm{O}(1)-\mathrm{C}(14)$ & $1.3472(19)$ & $\mathrm{C}(18)-\mathrm{C}(17)$ & $1.412(2)$ \\
\hline$C(20)-C(23)$ & $1.436(2)$ & $\mathrm{C}(18)-\mathrm{C}(21)$ & $1.435(2)$ \\
\hline $\mathrm{C}(20)-\mathrm{C}(19)$ & $1.449(2)$ & $\mathrm{C}(12)-\mathrm{N}(1)$ & $1.4286(19)$ \\
\hline $\mathrm{C}(19)-\mathrm{C}(18)$ & $1.414(2)$ & $\mathrm{C}(22)-\mathrm{C}(21)$ & $1.353(2)$ \\
\hline$C(19)-C(14)$ & $1.428(2)$ & $C(22)-C(23)$ & $1.426(2)$ \\
\hline$C(14)-C(15)$ & $1.399(2)$ & $\mathrm{N}(1)-\mathrm{C}(13)$ & $1.279(2)$ \\
\hline$C(15)-C(16)$ & $1.408(2)$ & $\mathrm{C}(16)-\mathrm{C}(17)$ & $1.372(2)$ \\
\hline$C(15)-C(13)$ & $1.464(2)$ & $\mathrm{C}(23)-\mathrm{C}(24)$ & $1.409(2)$ \\
\hline $\mathrm{N}(2)-\mathrm{C}(24)$ & $1.306(2)$ & & \\
\hline \multicolumn{4}{|c|}{ Angles } \\
\hline $\mathrm{O}(2)-\mathrm{C}(20)-\mathrm{C}(23)$ & $121.84(13)$ & $\mathrm{C}(14)-\mathrm{C}(15)-\mathrm{C}(13)$ & $119.49(14)$ \\
\hline $\mathrm{O}(2)-\mathrm{C}(20)-\mathrm{C}(19)$ & $120.39(13)$ & $\mathrm{C}(16)-\mathrm{C}(15)-\mathrm{C}(13)$ & $121.77(13)$ \\
\hline$C(23)-C(20)-C(19)$ & $117.76(14)$ & $\mathrm{C}(24)-\mathrm{N}(2)-\mathrm{C}(25)$ & $128.79(14)$ \\
\hline$C(18)-C(19)-C(14)$ & $119.48(13)$ & $\mathrm{C}(1)-\mathrm{C}(12)-\mathrm{N}(1)$ & $120.36(14)$ \\
\hline $\mathrm{C}(18)-\mathrm{C}(19)-\mathrm{C}(20)$ & $120.29(13)$ & $\mathrm{C}(8)-\mathrm{C}(12)-\mathrm{N}(1)$ & $117.93(14)$ \\
\hline$C(14)-C(19)-C(20)$ & $120.23(14)$ & $\mathrm{C}(13)-\mathrm{N}(1)-\mathrm{C}(12)$ & $116.33(13)$ \\
\hline $\mathrm{O}(1)-\mathrm{C}(14)-\mathrm{C}(15)$ & $119.10(14)$ & $\mathrm{N}(2)-\mathrm{C}(24)-\mathrm{C}(23)$ & $122.35(14)$ \\
\hline $\mathrm{O}(1)-\mathrm{C}(14)-\mathrm{C}(19)$ & $120.50(13)$ & $\mathrm{C}(26)-\mathrm{C}(25)-\mathrm{N}(2)$ & $118.65(14)$ \\
\hline$C(15)-C(14)-C(19)$ & $120.40(14)$ & $\mathrm{C}(33)-\mathrm{C}(25)-\mathrm{N}(2)$ & $117.90(14)$ \\
\hline$C(14)-C(15)-C(16)$ & $118.74(14)$ & $\mathrm{N}(1)-\mathrm{C}(13)-\mathrm{C}(15)$ & $122.81(15)$ \\
\hline
\end{tabular}


Table 3. Selected Bond Distances ( $\AA$ ) and Angles (deg) for compound 2.

\begin{tabular}{|c|c|c|c|}
\hline \multicolumn{4}{|c|}{ Distances } \\
\hline $\mathrm{C}(1)-\mathrm{N}(1)$ & $1.421(3)$ & $\mathrm{C}(22)-\mathrm{C}(23)$ & $1.412(3)$ \\
\hline $\mathrm{C}(13)-\mathrm{N}(1)$ & $1.274(3)$ & $\mathrm{C}(23)-\mathrm{C}(24)$ & $1.367(3)$ \\
\hline $\mathrm{C}(13)-\mathrm{C}(14)$ & $1.471(3)$ & $\mathrm{C}(24)-\mathrm{C}(25)$ & $1.415(3)$ \\
\hline $\mathrm{C}(14)-\mathrm{C}(17)$ & $1.396(3)$ & $C(25)-C(26)$ & $1.386(3)$ \\
\hline $\mathrm{C}(14)-\mathrm{C}(15)$ & $1.411(3)$ & $\mathrm{C}(25)-\mathrm{C}(30)$ & $1.470(3)$ \\
\hline $\mathrm{C}(15)-\mathrm{C}(16)$ & $1.356(3)$ & $\mathrm{C}(26)-\mathrm{O}(2)$ & $1.361(2)$ \\
\hline$C(16)-C(22)$ & $1.417(3)$ & $\mathrm{C}(27)-\mathrm{Si}(2)$ & $1.857(3)$ \\
\hline $\mathrm{C}(17)-\mathrm{O}(1)$ & $1.370(2)$ & $\mathrm{C}(28)-\mathrm{Si}(2)$ & $1.843(3)$ \\
\hline $\mathrm{C}(17)-\mathrm{C}(21)$ & $1.427(3)$ & $\mathrm{C}(29)-\mathrm{Si}(2)$ & $1.851(3)$ \\
\hline $\mathrm{C}(18)-\mathrm{Si}(1)$ & $1.856(2)$ & $\mathrm{C}(30)-\mathrm{N}(2)$ & $1.262(3)$ \\
\hline $\mathrm{C}(19)-\mathrm{Si}(1)$ & $1.846(2)$ & $\mathrm{C}(31)-\mathrm{N}(2)$ & $1.425(2)$ \\
\hline $\mathrm{C}(20)-\mathrm{Si}(1)$ & $1.849(2)$ & $\mathrm{O}(1)-\mathrm{Si}(1)$ & $1.6822(15)$ \\
\hline $\mathrm{C}(21)-\mathrm{C}(22)$ & $1.426(3)$ & $\mathrm{O}(2)-\mathrm{Si}(2)$ & $1.6765(15)$ \\
\hline$C(21)-C(26)$ & $1.441(2)$ & & \\
\hline \multicolumn{4}{|c|}{ Angles } \\
\hline $\mathrm{C}(9)-\mathrm{C}(1)-\mathrm{N}(1)$ & $117.48(17)$ & $\mathrm{O}(2)-\mathrm{C}(26)-\mathrm{C}(21)$ & $120.45(17)$ \\
\hline $\mathrm{C}(2)-\mathrm{C}(1)-\mathrm{N}(1)$ & $120.93(18)$ & $\mathrm{C}(25)-\mathrm{C}(26)-\mathrm{C}(21)$ & $120.67(16)$ \\
\hline $\mathrm{N}(1)-\mathrm{C}(13)-\mathrm{C}(14)$ & $120.58(18)$ & $\mathrm{N}(2)-\mathrm{C}(30)-\mathrm{C}(25)$ & $121.31(17)$ \\
\hline $\mathrm{C}(17)-\mathrm{C}(14)-\mathrm{C}(15)$ & $119.24(17)$ & $\mathrm{C}(32)-\mathrm{C}(31)-\mathrm{C}(39)$ & $121.03(17)$ \\
\hline $\mathrm{C}(17)-\mathrm{C}(14)-\mathrm{C}(13)$ & $121.30(17)$ & $\mathrm{C}(32)-\mathrm{C}(31)-\mathrm{N}(2)$ & $119.48(18)$ \\
\hline $\mathrm{C}(15)-\mathrm{C}(14)-\mathrm{C}(13)$ & $119.44(17)$ & $\mathrm{C}(39)-\mathrm{C}(31)-\mathrm{N}(2)$ & $119.21(17)$ \\
\hline$C(16)-C(15)-C(14)$ & $121.30(18)$ & $\mathrm{C}(13)-\mathrm{N}(1)-\mathrm{C}(1)$ & $121.56(18)$ \\
\hline$C(15)-C(16)-C(22)$ & $120.59(18)$ & $\mathrm{C}(30)-\mathrm{N}(2)-\mathrm{C}(31)$ & $120.28(17)$ \\
\hline $\mathrm{O}(1)-\mathrm{C}(17)-\mathrm{C}(14)$ & $118.46(17)$ & $\mathrm{C}(17)-\mathrm{O}(1)-\mathrm{Si}(1)$ & $124.56(12)$ \\
\hline $\mathrm{O}(1)-\mathrm{C}(17)-\mathrm{C}(21)$ & $120.89(16)$ & $\mathrm{C}(26)-\mathrm{O}(2)-\mathrm{Si}(2)$ & $127.82(12)$ \\
\hline $\mathrm{C}(14)-\mathrm{C}(17)-\mathrm{C}(21)$ & $120.64(17)$ & $\mathrm{O}(1)-\mathrm{Si}(1)-\mathrm{C}(19)$ & $104.29(10)$ \\
\hline $\mathrm{C}(22)-\mathrm{C}(21)-\mathrm{C}(17)$ & $117.73(16)$ & $\mathrm{O}(1)-\mathrm{Si}(1)-\mathrm{C}(20)$ & $111.42(9)$ \\
\hline $\mathrm{C}(22)-\mathrm{C}(21)-\mathrm{C}(26)$ & $117.54(17)$ & $\mathrm{C}(19)-\mathrm{Si}(1)-\mathrm{C}(20)$ & $110.55(12)$ \\
\hline $\mathrm{C}(17)-\mathrm{C}(21)-\mathrm{C}(26)$ & $124.72(17)$ & $\mathrm{O}(1)-\mathrm{Si}(1)-\mathrm{C}(18)$ & $109.54(9)$ \\
\hline $\mathrm{C}(23)-\mathrm{C}(22)-\mathrm{C}(16)$ & $120.16(17)$ & $\mathrm{C}(19)-\mathrm{Si}(1)-\mathrm{C}(18)$ & $111.01(12)$ \\
\hline $\mathrm{C}(23)-\mathrm{C}(22)-\mathrm{C}(21)$ & $120.11(17)$ & $\mathrm{C}(20)-\mathrm{Si}(1)-\mathrm{C}(18)$ & $109.92(11)$ \\
\hline$C(16)-C(22)-C(21)$ & 119.73(18) & $\mathrm{O}(2)-\mathrm{Si}(2)-\mathrm{C}(28)$ & $102.82(11)$ \\
\hline $\mathrm{C}(24)-\mathrm{C}(23)-\mathrm{C}(22)$ & $120.51(18)$ & $\mathrm{O}(2)-\mathrm{Si}(2)-\mathrm{C}(29)$ & $109.12(11)$ \\
\hline $\mathrm{C}(23)-\mathrm{C}(24)-\mathrm{C}(25)$ & $121.04(18)$ & $\mathrm{C}(28)-\mathrm{Si}(2)-\mathrm{C}(29)$ & $109.70(15)$ \\
\hline $\mathrm{C}(26)-\mathrm{C}(25)-\mathrm{C}(24)$ & $119.60(17)$ & $\mathrm{O}(2)-\mathrm{Si}(2)-\mathrm{C}(27)$ & $111.30(11)$ \\
\hline$C(26)-C(25)-C(30)$ & $121.15(16)$ & $\mathrm{C}(28)-\mathrm{Si}(2)-\mathrm{C}(27)$ & $115.21(17)$ \\
\hline $\mathrm{C}(24)-\mathrm{C}(25)-\mathrm{C}(30)$ & $119.25(17)$ & $\mathrm{C}(29)-\mathrm{Si}(2)-\mathrm{C}(27)$ & $108.49(14)$ \\
\hline $\mathrm{O}(2)-\mathrm{C}(26)-\mathrm{C}(25)$ & $118.88(16)$ & & \\
\hline
\end{tabular}

